\title{
Eudesmanolides and Further Terpenes from the Leaves of Eupatorium semialatum ${ }^{\mathrm{d}}$
}

\author{
Günter Langa , Claus M. Passreiter ${ }^{\mathrm{a}, *}$, Beatriz E. Medinilla ${ }^{\mathrm{b}}$ and Juan-José Castillo ${ }^{\mathrm{c}}$ \\ ${ }^{a}$ Institut für Pharmazeutische Biologie, Heinrich-Heine-Universität, Universitätsstr. 1, \\ D-40225 Düsseldorf, Germany. Fax: +49 2118111923 , E-mail: passreit@uni-duesseldorf.de \\ b Facultad Ciencias Químicas y Farmacía, Universidad de San Carlos, Guatemala Ciudad, \\ Guatemala \\ c Facultad de Agronomía, Universidad de San Carlos, Guatemala Ciudad, Guatemala \\ ${ }^{d}$ Part of a current dissertation of G. Lang, Heinrich-Heine-Universität, Düsseldorf \\ * Author for correspondence and reprint requests \\ Z. Naturforsch., 55c, 511-515 (2000); received March 23, 2000
}

Eupatorium semialatum, Sesquiterpene Lactones, Eudesmanolides

\begin{abstract}
A collection of Eupatorium semialatum from Guatemala was investigated for the occurrence of sesquiterpene lactones. In addition to the sesquiterpene cinnamoyloxyoplopanone (5), lactones of the eudesmanolide type were found and identified by NMR as derivatives of $8 \beta$-hydroxyreynosin and $8 \beta$-hydroxybalchanin, esterified with 2 -substituted acrylic acids. The acid moieties were determined to be the enantiomeric $(R)$ - and $(S)$-(2-hydroxy-1-methoxyethyl)acrylic acid, in 1, 2 and $\mathbf{4}$ respectively, and (1-hydroxy-ethyl)acrylic acid in $\mathbf{3}$.
\end{abstract}

\section{Introduction}

Eupatorium semialatum Benth. (Asteraceae) occurs in tropical areas of Guatemala and Honduras (Clewell, 1975; Nash and Williams, 1976). A bitter decoction prepared from its leaves is a popular remedy in the Alta Verapaz of Guatemala for treatment of malaria, diabetes, amoebiasis, hepatitis, stomach and intestinal inflammation in humans and for treatment of colic in domestic animals (Cáceres, 1996; Medinilla, 1978; Morton, 1981).

Whereas Nash and Williams (1976) describe the Guatemalan E. semialatum to be quite different from the South Mexican E. ligustrinum, King and Robinson (1987) treated both as a single species in the recent reorganization of several genera of the tribe Eupatorieae. Moreover, as a result of their studies, E. ligustrinum was suggested to belong to the genus Ageratina (King and Robinson, 1970). This species (A. ligustrina) was previously investigated for the occurrence of sesquiterpene lactones (Romo et al., 1968; Tamayo-Castillo et al., 1988), but no information exists about the secondary metabolites of E. semialatum.

In continuation of our phytochemical investigations on antimalarial plants from Guatemala we now report new $(\mathbf{1}, \mathbf{2}$ and $\mathbf{4})$ and known terpenoids $(\mathbf{3}, \mathbf{5}$ and $\mathbf{6})$ from the leaves of E. semialatum.

\section{Results and Discussion}

Purification of the methanol soluble phase of the dichloromethane extract afforded two fractions containing the sesquiterpene lactones 1-4, the oplopanone derivative $\mathbf{5}$, as well as oleanolic acid 6 (Mahato and Kundu, 1994). Their structures were elucidated after purification using $\mathrm{CC}$, preparative TLC and preparative HPLC by NMR, including 2D-COSY, HMQC, and HMBC experiments.

Compounds $\mathbf{1}$ and $\mathbf{2}$ were isolated as a mixture. All attempts to separate them by repeated HPLC using different solvent systems failed. Surprisingly, we found only one peak in GLC. In the simultaneously recorded mass spectrum the molecular ion was found at $m / z 264$, a molecular weight often found for sesquiterpene lactones (Seaman, 1982). The additionally recorded DCI-MS displayed the base ion at $\mathrm{m} / z 410\left[\mathrm{M}+\mathrm{NH}_{4}\right]^{+}$, which makes it obvious that a degradation occurs most likely in the injector of the GC, which for both compounds leads to the same product $\left([\mathrm{M}]^{+}\right.$at $\left.m / z 264\right)$ by the loss of a fragment with a relative mass of $\mathrm{M}_{\mathrm{r}} 128$.

The ${ }^{13} \mathrm{C}$ NMR spectrum displayed thirty-two signals corresponding to forty-two carbons, at shift values characteristic for eudesmanolide ester derivatives (Budesinsky and Saman, 1995). Whereas ten signals represented two carbons each, as seen 


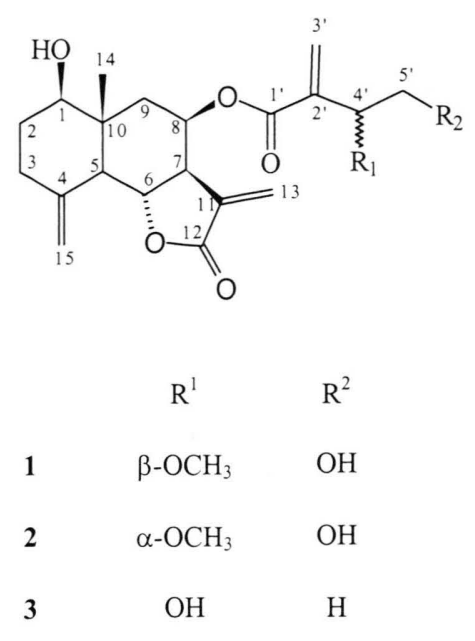<smiles>C=C(C(=O)OC1C[C@]2(C)C(O)CC=C(C)C2[C@H]2OC(=O)C(=C)[C@H]12)[C@@H](CO)OC</smiles><smiles>CC(C)C1CC[C@H](C)[C@H]2CCC(C(=O)COC(=O)/C=C/c3ccccc3)[C@H]12</smiles>

by the 2D-HMQC and HMBC spectra, twentytwo of them were found in pairs with shift differences between the analogous signals of only 0.02 to $0.12 \mathrm{ppm}$. Main differences existed for carbons C-7, C-8, C-9 and C-13 (see Experimental Section). From the shift values of C-1-C-15 of $\mathbf{1}$ and 2 in accord with the literature (Budesinsky and Saman, 1995) it was obvious that the alcohol moiety consisted of $8 \beta$-hydroxyreynosin in both cases. Differences between the two compounds exist only in the acid side chain. The remaining signals, due to two similar acid moieties, suggested the presence of a 2-substituted acrylic acid, which additionally contained one methine-, one hydroxymethylene- and one methoxy-group. This means that the side chain at C-2 consists of a 2-hydroxy1-methoxyethyl-group in both cases (Budesinsky and Saman, 1987).

As expected, the corresponding signals for two nearly identical reynosin derivatives were found in the ${ }^{1} \mathrm{H}$ NMR spectrum. Except for a few (see Experimental Section), most signals overlapped. Since all coupling constants from $\mathbf{1}$ and $\mathbf{2}$ were found in the same range, their relative stereochemistry should be identical. The position of the esterification at C-8 clearly followed from the shift values of the respective signals and the couplings found in the COSY spectrum. Shift differences between the signals of $\mathrm{H}-8$ and $\mathrm{H}-13$ in the ${ }^{1} \mathrm{H}$ NMR of $\mathbf{1}$ and $\mathbf{2}$, respectively, suggested that the at-

tached acids should be different, but the 2DCOSY, HMBC and HMQC spectra clearly indicated that the constitutions of both acids are the same. Therefore, both acids can only differ in the absolute configuration at C-4', although direct proof is missing. Hence, $\mathbf{1}$ and $\mathbf{2}$ are diastereomeric esters built from $8 \beta$-hydroxyreynosin with the enantiomeric 4'(R)- and 4'(S)-(2-hydroxy-1-methoxyethyl)acrylic acid. Unfortunately, we could not determine, which particular acid was attached to $\mathbf{1}$ and $\mathbf{2}$, respectively, since all attempts to separate the compounds and subsequently obtain crystals for X-ray analysis were unsuccessful.

Compound $\mathbf{3}$ was identified as another ester derivative of $8 \beta$-hydroxyreynosin, differing from $\mathbf{1}$ and $\mathbf{2}$ only by its ester side chain (Budesinsky and Saman, 1995). This compound was previously isolated by Ober et al. (1994) from Calea trichomata, but due to overlapping signals in their $200 \mathrm{MHz}$ NMR spectra, they were not able to assign the signals for $\mathrm{H}-2$ and $\mathrm{H}-3$, respectively. Because of the higher resolution of our spectra and through comparison to $\mathbf{1}$ and $\mathbf{2}$, we were now able to assign the four separated signals in the ${ }^{1} \mathrm{H}$ NMR $(\mathrm{H}-2$ : $\delta 1.85$ $\mathrm{m}$ and $1.62 \mathrm{~m} ; \mathrm{H}-3: \delta 2.37 \mathrm{~m}$ and $2.17 \mathrm{~m}$ ).

Compound $\mathbf{4}$ was isolated as a pure gum in small quantity. Its mass and NMR spectra indicated that $\mathbf{4}$ is an isomer of $\mathbf{1}$ and $\mathbf{2}$, differing in the sesquiterpene lactone moiety. Instead of the signals for the exocyclic methylene group (H-15) and the methy- 
lene group (H-3) in $\mathbf{1 - 3}$, we found a three-proton singlet and a multiplet for one proton at shift values characteristic for a eudesmanolide containing a $\Delta 3,4$ double bond. This was confirmed by the corresponding signals in the ${ }^{13} \mathrm{C}$ NMR spectrum. Compound 4 was therefore identified as a $8 \beta$-acyloxy derivative of balchanin (Budesinsky and Saman, 1995), esterified with one of the enantiomeric (2-hydroxy-1-methoxyethyl)acrylic acids.

GLC/MS and NMR spectra indicated the structure of compound $\mathbf{5}$ as 11-cinnamoyloxyoplopanone, which was previously found in Ageratina ligustrina (Tamayo-Castillo et al., 1988), but the reported ${ }^{1} \mathrm{H}$ NMR data were incomplete and ${ }^{13} \mathrm{C}$ NMR data were missing. The correct assignments of the complete NMR signals, confirmed by 2DCOSY, 2D-HMQC and 2D-HMBC, are given here for the first time (see Experimental Section).

The triterpene oleanolic acid (6) was identified by interpretation of its NMR data in comparison to an authentic sample (Mahato and Kundu, 1994).

Previous investigations have shown that collections of A. ligustrina (=E. ligustrinum) from Mexico, contain sesquiterpene lactones of the guaianolide and germacranolide type (Romo et al., 1968; Tamayo-Castillo et al., 1988). The sesquiterpene lactones found in our plant material from Guatemala are of the eudesmanolide type. Although we intensively searched for compounds previously reported to occur in A. ligustrina, the only constituent which occurs in both species, was the oplopanone sesquiterpene 5. Therefore it seems very likely that E. semialatum is in fact a different species, as already suggested by Nash in the Flora of Guatemala (Nash and Williams, 1976), and not identical to A. ligustrina or E. ligustrinum. Further investigations on the sesquiterpene lactone pattern of E. semialatum are in progress.

\section{Experimental Section}

\section{General experimental procedures}

NMR: Bruker DRX 500, $500 \mathrm{MHz}\left({ }^{1} \mathrm{H}\right.$ NMR) and $125 \mathrm{MHz}\left({ }^{13} \mathrm{C} \mathrm{NMR}\right)$ in $\mathrm{CDCl}_{3}$ or acetone- $\mathrm{d}_{6}$, respectively, or Bruker DMX-600, $600 \mathrm{MHz}\left({ }^{1} \mathrm{H}\right.$ $\mathrm{NMR})$ and $150 \mathrm{MHz}\left({ }^{13} \mathrm{C} \mathrm{NMR}\right)$ in $\mathrm{CDCl}_{3}$. GCMS: EI $(70 \mathrm{eV})$ using the GC-MS mode on a MSD 5972 combined with a 5890 plus gas chromatograph (Hewlett-Packard); column $25 \mathrm{~m} \times 0.25 \mathrm{~mm}$ (Optima-1, Macherey \& Nagel). Temperature pro- gression: $150{ }^{\circ} \mathrm{C}(3 \mathrm{~min})$ to $280{ }^{\circ} \mathrm{C}$ at $10^{\circ} \mathrm{min}^{-1}$. MS: EI $(70 \mathrm{eV})$ direct inlet on a Finnigan MAT INCOS 50 at $200{ }^{\circ} \mathrm{C}$. HPLC: HP 1050 system, equipped with DAD detector. Detector channels set at 215 and $260 \mathrm{~nm}$, with a $\mathrm{RP}_{18}$ Hypersil ODS (5 $\mu \mathrm{m})$ column $(125 \times 5 \mathrm{~mm})$. Mobile phase: 1-3: $\mathrm{MeOH}-\mathrm{H}_{2} \mathrm{O}(2: 3 \mathrm{v} / \mathrm{v})$ to $\mathrm{MeOH}-\mathrm{H}_{2} \mathrm{O}(11: 9 \mathrm{v} / \mathrm{v})$ at $1.0 \mathrm{ml} \mathrm{min}{ }^{-1}$ for $20 \mathrm{~min} ; 4: \mathrm{MeOH}-\mathrm{H}_{2} \mathrm{O}(1: 4$ $\mathrm{v} / \mathrm{v})$ to $\mathrm{MeOH}(100 \%)$, at $1.0 \mathrm{ml} \mathrm{min}^{-1}$ for $60 \mathrm{~min}$; 5: $\mathrm{MeOH}-\mathrm{H}_{2} \mathrm{O}(3: 1 \mathrm{v} / \mathrm{v})$ to $\mathrm{MeOH}-\mathrm{H}_{2} \mathrm{O}$ (4:1 $\mathrm{v} / \mathrm{v})$ at $1.0 \mathrm{ml} \mathrm{min}^{-1}$ for $7 \mathrm{~min}$. TLC: Si gel $60 \mathrm{~F}_{254}$ (Merck) $\mathrm{CH}_{2} \mathrm{Cl}_{2} / \mathrm{MeOH}(2: 1 \mathrm{v} / \mathrm{v})$. Detection with anisealdehyde $/ \mathrm{H}_{2} \mathrm{SO}_{4}$.

\section{Plant material}

Leaves of Eupatorium semialatum Benth. (725 g), collected near Purulhá, Baja Verapaz, Guatemala, were identified by one of us (J. J. C.). Vouchers (JC2541) are deposited at the Facultad de Agronomía, Universidad de San Carlos de Guatemala.

\section{Isolation}

Dried and powdered leaves $(725 \mathrm{~g})$ were extracted with $\mathrm{CH}_{2} \mathrm{Cl}_{2}$ in a Soxhlet apparatus. The extract $(81 \mathrm{~g})$ was treated with $\mathrm{MeOH}$ to obtain $36.86 \mathrm{~g}$ of $\mathrm{MeOH}$ solubles. This extract was purified by repeated CC on Sephadex LH-20 with $\mathrm{MeOH}$, followed by $\mathrm{CC}$ on silica gel 60 with $\mathrm{CH}_{2} \mathrm{Cl}_{2} / \mathrm{MeOH}(9: 1 \mathrm{v} / \mathrm{v})$ and Sephadex LH-20 with cyclohexane/ $\mathrm{CH}_{2} \mathrm{Cl}_{2} / \mathrm{MeOH}(7: 4: 1 \mathrm{v} / \mathrm{v})$. The fractions obtained were further purified by preparative TLC using $\mathrm{CH}_{2} \mathrm{Cl}_{2} / \mathrm{MeOH}(2: 1 \mathrm{v} / \mathrm{v})$ as the solvent system and preparative HPLC (in $0.5 \mathrm{mg}$ aliquots) to give a mixture of $\mathbf{1}$ and $\mathbf{2}(3 \mathrm{mg})$ and the pure compounds $3(1 \mathrm{mg}), \mathbf{4}(0.5 \mathrm{mg})$, and $\mathbf{5}$ (4 mg). Compound 6 (20 mg) was isolated by preparative TLC.

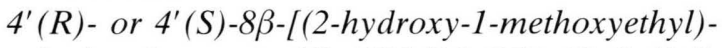
acryloyloxy]reynosin (1): UV $\left[\mathrm{MeOH}-\mathrm{H}_{2} \mathrm{O}(1: 1)\right.$ $\mathrm{v} / \mathrm{v}] \lambda_{\max } 210 \mathrm{~nm} ;{ }^{1} \mathrm{H} \mathrm{NMR}\left(\mathrm{CDCl}_{3}, 500 \mathrm{MHz}\right)$

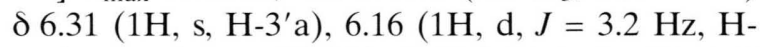
13a), $5.94\left(1 \mathrm{H}, \mathrm{s}, \mathrm{H}-3^{\prime} \mathrm{b}\right), 5.85(1 \mathrm{H}, \mathrm{d}, J=2.5,3.8$ $\mathrm{Hz}, \mathrm{H}-8), 5.45$ (1H, d, $J=3.2 \mathrm{~Hz}, \mathrm{H}-13 \mathrm{~b}), 5.04$ (1H, s, H-15a), 4.97 (1H, s, H-15b), $4.52(1 \mathrm{H}, \mathrm{t}, J=$ 11.1, Hz, H-6), 4.21 (1H, dd, $\left.J=2.5,6.9 \mathrm{~Hz}, \mathrm{H}-4^{\prime}\right)$, 3.69 (1H, m, H-5'a), 3.53 (1H, dd, $J=4.4,11.3 \mathrm{~Hz}$, $\mathrm{H}-1), 3.48\left(1 \mathrm{H}, \mathrm{m}, \mathrm{H}-5^{\prime} \mathrm{b}\right), 3.33\left(3 \mathrm{H}, \mathrm{s},-\mathrm{OCH}_{3}\right)$, $2.86(1 \mathrm{H}, \mathrm{ddd}, J=3.2,5.7,11.1 \mathrm{~Hz}, \mathrm{H}-7), 2.42(1 \mathrm{H}$, 
$\mathrm{dd}, J=2.5,15.1 \mathrm{~Hz}, \mathrm{H}-9 \mathrm{a}), 2.36(1 \mathrm{H}, \mathrm{m}, \mathrm{H}-3 \mathrm{a})$ $2.27(1 \mathrm{H}, \mathrm{d}, J=10.7, \mathrm{H}-5), 2.15(1 \mathrm{H}, \mathrm{m}, \mathrm{H}-3 \mathrm{~b})$, $1.84(1 \mathrm{H}, \mathrm{m}, \mathrm{H}-2 \mathrm{a}), 1.66(1 \mathrm{H}, \mathrm{dd}, J=3.8,15.1 \mathrm{~Hz}$, $\mathrm{H}-9 \mathrm{~b}), 1.60(1 \mathrm{H}, \mathrm{m}, \mathrm{H}-2 \mathrm{~b}), 1.00(3 \mathrm{H}, \mathrm{s}, \mathrm{H}-14) ;{ }^{13} \mathrm{C}$ NMR $\left(\mathrm{CDCl}_{3}, 125 \mathrm{MHz}\right) \delta 169.54(\mathrm{C}-12), 165.14$ (C-1'), 141.61 (C-2'), 136.69 (C-4), 134.32 (C-11), 127.51 (C-3'), 119.56 (C-13), 111.00 (C-15), 80.33 (C-4'), 78.45 (C-1), 74.94 (C-6), 66.64 (C-8), 65.30 $\left(\mathrm{C}-5^{\prime}\right), 57.29\left(-\mathrm{OCH}_{3}\right), 53.31(\mathrm{C}-5), 51.99(\mathrm{C}-7)$, 42.57 (C-10), 40.32 (C-9), 33.26 (C-3), 30.92 (C-2), 13.68 (C-14); EIMS m/z 264 (1), 246 (7), 228 (11), 213 (5), 202 (13), 179 (34), 161 (24), 145 (13), 133 (22), 117 (19), 106 (52), 91 (64), 79 (51), 69 (58), 55 (44), 41 (100).

$4^{\prime}(S)$ - or $4^{\prime}(R)-8 \beta-[(2-h y d r o x y-1-m e t h o x y e t h y l)$ acryloyloxy]reynosin (2): UV [ $\mathrm{MeOH}-\mathrm{H}_{2} \mathrm{O}(1: 1)$ $\mathrm{v} / \mathrm{v}] \lambda_{\max } 211 \mathrm{~nm} ;{ }^{1} \mathrm{H}$ NMR $\left(\mathrm{CDCl}_{3}, 500 \mathrm{MHz}\right)$ d $6.32\left(1 \mathrm{H}, \mathrm{s}, \mathrm{H}-3^{\prime} \mathrm{a}\right), 6.17(1 \mathrm{H}, \mathrm{d}, J=3.2 \mathrm{~Hz}, \mathrm{H}-$ 13a), $5.93\left(1 \mathrm{H}, \mathrm{s}, \mathrm{H}-3^{\prime} \mathrm{b}\right), 5.82(1 \mathrm{H}, \mathrm{d}, J=2.5,3.8$ $\mathrm{Hz}, \mathrm{H}-8), 5.46(1 \mathrm{H}, \mathrm{d}, J=3.2 \mathrm{~Hz}, \mathrm{H}-13 \mathrm{~b}), 5.04$ (1H, s, H-15a), 4.97 (1H, s, H-15b), $4.52(1 \mathrm{H}, \mathrm{t}, J=$ $11.1, \mathrm{~Hz}, \mathrm{H}-6), 4.21\left(1 \mathrm{H}, \mathrm{dd}, J=2.5,6.9 \mathrm{~Hz}, \mathrm{H}-4^{\prime}\right)$, $3.69\left(1 \mathrm{H}, \mathrm{m}, \mathrm{H}-5^{\prime} \mathrm{a}\right), 3.53(1 \mathrm{H}, \mathrm{dd}, J=4.4,11.3 \mathrm{~Hz}$, $\mathrm{H}-1), 3.48\left(1 \mathrm{H}, \mathrm{m}, \mathrm{H}-5^{\prime} \mathrm{b}\right), 3.34\left(3 \mathrm{H}, \mathrm{s},-\mathrm{OCH}_{3}\right)$, $2.86(1 \mathrm{H}$, ddd, $J=3.2,5.7,11.1 \mathrm{~Hz}, \mathrm{H}-7), 2.43(1 \mathrm{H}$, $\mathrm{dd}, J=2.5,15.1 \mathrm{~Hz}, \mathrm{H}-9 \mathrm{a}), 2.36(1 \mathrm{H}, \mathrm{m}, \mathrm{H}-3 \mathrm{a})$, $2.27(1 \mathrm{H}, \mathrm{d}, J=10.7, \mathrm{H}-5), 2.15(1 \mathrm{H}, \mathrm{m}, \mathrm{H}-3 \mathrm{~b})$, $1.84(1 \mathrm{H}, \mathrm{m}, \mathrm{H}-2 \mathrm{a}), 1.66(1 \mathrm{H}, \mathrm{dd}, J=3.8,15.1 \mathrm{~Hz}$, $\mathrm{H}-9 \mathrm{~b}), 1.60(1 \mathrm{H}, \mathrm{m}, \mathrm{H}-2 \mathrm{~b}), 0.98(3 \mathrm{H}, \mathrm{s}, \mathrm{H}-14) ;{ }^{13} \mathrm{C}$ NMR $\left(\mathrm{CDCl}_{3}, 125 \mathrm{MHz}\right)$ o 169.54 (C-12), 165.09 (C-1'), 141.61 (C-2'), 136.71 (C-4), 134.32 (C-11), 127.54 (C-3'), 119.47 (C-13), 110.97 (C-15), 80.33 (C-4'), 78.45 (C-1), 74.99 (C-6), 66.73 (C-8), 65.26 $\left(\mathrm{C}-5^{\prime}\right), 57.31\left(-\mathrm{OCH}_{3}\right), 53.31(\mathrm{C}-5), 51.92(\mathrm{C}-7)$, 42.57 (C-10), 40.20 (C-9), 33.26 (C-3), 30.90 (C-2), 13.68 (C-14); EIMS m/z 264 (1), 246 (7), 228 (11), 213 (5), 202 (13), 179 (34), 161 (24), 145 (13), 133 (22), 117 (19), 106 (52), 91 (64), 79 (51), 69 (58), 55 (44), 41 (100).

$8 \beta$-[(2-hydroxy-1-methoxyethyl)acryloyloxy]balchanin (4): UV $\left[\mathrm{MeOH}-\mathrm{H}_{2} \mathrm{O}\right.$ (11:9) v/v] $\lambda_{\max }$ $218 \mathrm{~nm} ;{ }^{1} \mathrm{H}$ NMR (acetone- $\left.\mathrm{d}_{6}, 500 \mathrm{MHz}\right) \delta 6.35$ (1H, s, H-3'a ), 5.99 (1H, d, J = 3.2 Hz, H-13a), 5.87 $\left(1 \mathrm{H}, \mathrm{s}, \mathrm{H}-3^{\prime} \mathrm{b}\right), 5.80(1 \mathrm{H}, \mathrm{dd}, J=2.5,3.2 \mathrm{~Hz}, \mathrm{H}-8)$, $5.41(1 \mathrm{H}, \mathrm{d}, J=3.2 \mathrm{~Hz}, \mathrm{H}-13 \mathrm{~b}), 5.35(1 \mathrm{H}, \mathrm{m}, \mathrm{H}-$ 3), $4.52(1 \mathrm{H}, \mathrm{t}, J=10.7 \mathrm{~Hz}, \mathrm{H}-6), 4.18(1 \mathrm{H}, \mathrm{dd}, J=$ $\left.3.2,6.9 \mathrm{~Hz}, \mathrm{H}-4^{\prime}\right), 3.66(1 \mathrm{H}, \mathrm{m}, \mathrm{H}-1), 3.57(1 \mathrm{H}, \mathrm{m}$, H-5'a), $3.40\left(1 \mathrm{H}, \mathrm{m}, \mathrm{H}-5^{\prime} \mathrm{b}\right), 3.29\left(3 \mathrm{H}, \mathrm{s},-\mathrm{OCH}_{3}\right)$, $3.06(1 \mathrm{H}, \mathrm{m}, J=5.7 \mathrm{~Hz}, \mathrm{H}-7), 2.55(1 \mathrm{H}, \mathrm{d}, J=9.5$ $\mathrm{Hz}, \mathrm{H}-5), 2.37$ (1H, dd, $J=15.1 \mathrm{~Hz}, \mathrm{H}-9 \mathrm{a}), 2.28$
(1H, m, H-2a), 1.96 (1H, m, H-2b), 1.83 (3H, s, H15), $1.71(1 \mathrm{H}, \mathrm{dd}, J=3.2,15.1 \mathrm{~Hz}, \mathrm{H}-9 \mathrm{~b}), 1.07$ $(3 \mathrm{H}, \mathrm{s}, \mathrm{H}-14) ;{ }^{13} \mathrm{C}$ NMR (acetone- $\mathrm{d}_{6}, 125 \mathrm{MHz}$ ) ठ 169.42 (C-12), 165.59 (C-1'), 145.28 (C-2'), 138.53 (C-11), 133.32 (C-4), 126.63 (C-3'), 122.31 (C-3), 117.75 (C-13), 81.42 (C-4'), 77.60 (C-6), 74.85 (C-1), 67.42 (C-8), 65.22 (C-5'), 56.82 $\left(-\mathrm{OCH}_{3}\right), 53.18(\mathrm{C}-7), 51.15(\mathrm{C}-5), 40.88(\mathrm{C}-10)$, 39.30 (C-9), 32.02 (C-2), 22.76 (C-15), 13.80 (C14); EIMS $m / z, 264$ (14), 246 (2), 228 (3), 217 (6), 202 (5), 189 (16), 175 (9), 173 (9), 164 (25), 150 (17), 119 (39), 97 (34), 91 (61), 79 (45), 69 (64), 55 (50), 43 (59), 41 (100).

11-cinnamoyloxyoplopanone (5): UV [ $\mathrm{MeOH}-$ $\left.\mathrm{H}_{2} \mathrm{O}(4: 1) \mathrm{v} / \mathrm{v}\right] \lambda_{\max } 282 \mathrm{~nm} ;{ }^{1} \mathrm{H}$ NMR $\left(\mathrm{CDCl}_{3}, 600\right.$ $\mathrm{MHz}) \delta 7.76\left(1 \mathrm{H}, \mathrm{d}, J=16 \mathrm{OHz}, \mathrm{H}-3^{\prime}\right), 7.53(2 \mathrm{H}$, m, H-6', H-8' ), 7.39 (3H, m, H-5', H-7', H-9' ), 6.53 $\left(1 \mathrm{H}, \mathrm{d}, J=16.0 \mathrm{~Hz}, \mathrm{H}-2^{\prime}\right), 4.89(1 \mathrm{H}, \mathrm{d}, J=16.9$ Hz, H-11a), 4.80 (1H, d, $J=16.9 \mathrm{~Hz}, \mathrm{H}-11 \mathrm{~b}), 2.70$ $(1 \mathrm{H}$, ddd, $J=5.3,9.4,11.7 \mathrm{~Hz}, \mathrm{H}-3), 1.98(1 \mathrm{H}, \mathrm{m}$, $\mathrm{H}-2 \mathrm{a}), 1.95$ (1H, m, H-4), 1.83 (1H, m, H-1a), 1.79 $(1 \mathrm{H}, \mathrm{m}, \mathrm{H}-7 \mathrm{a}), 1.71(1 \mathrm{H}, \mathrm{m}, \mathrm{H}-2 \mathrm{~b}), 1.60(1 \mathrm{H}, \mathrm{m}$, H-6a), $1.51(1 \mathrm{H}, \mathrm{m}, \mathrm{H}-9), 1.45$ (1H, m, H-1b), 1.42 $(1 \mathrm{H}, \mathrm{m}, \mathrm{H}-12), 1.39$ (1H, m, H-7b), 1.19 (3H, s, H15), $1.09(1 \mathrm{H}, \mathrm{m}, \mathrm{H}-5), 1.08$ (1H, m, H-6b), 0.91 $(3 \mathrm{H}, \mathrm{d}, J=6.8 \mathrm{~Hz}, \mathrm{H}-13), 0.69(3 \mathrm{H}, \mathrm{d}, J=6.8 \mathrm{~Hz}$, $\mathrm{H}-14) ;{ }^{13} \mathrm{C}$ NMR $\left(\mathrm{CDCl}_{3}, 150 \mathrm{MHz}\right) \delta 206.19$ (C-10), 166.07 (C-1'), 146.12 (C-3'), 134.27 (C-4'),

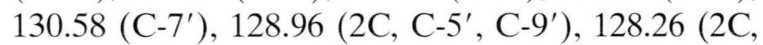
C-6', C-8'), 116.98 (C-2'), 73.09 (C-8), 67.81 (C11), 57.06 (C-9), 50.77 (C-3), 49.37 (C-5), 46.30 (C-4), 42.05 (C-7), 29.92 (C-12), 29.12 (C-2), 25.48 (C-1), 23.01 (C-6), 22.02 (C-13), 20.38 (C-15), 15.56 (C-14); EIMS $m / z 384[\mathrm{M}]^{+}(0.5), 382(0.5)$, 299 (1), 236 (9), 223 (17), 187 (15), 177 (30), 147 (6), 131 (100), 121 (14), 103 (42), 95 (16), 93 (9), 91 (9), 81 (14), 77 (26), 71 (15), 69 (11), 67 (11), 55 (11), 43 (40), 41 (12).

\section{Acknowledgements}

We are grateful to Dr. W. Peters and service staff, Institut für Anorganische Chemie und Strukturchemie, Universität Düsseldorf, for recording the $500 \mathrm{MHz}$ NMR spectra, and Dr. U. Matthiesen, Institut für Klinische Chemie und Laboratoriumsdiagnostik, Universität Düsseldorf, for taking the direct inlet-MS spectra. We thank Prof. Dr. M. B. Isman, Agricultural Sciences, University of British Columbia, Vancouver, Canada for reviewing the manuscript prior to submisson. 
Budesinsky M. and Saman D. (1987), Identification of acyl goups ocurring in sesquiterpene lactones: Proton and carbon-13 NMR study. Collect. Czech. Chem Commun. 52, 453-475.

Budesinsky M. and Saman D. (1995), Carbon-13 NMR spectra of sesquiterpene lactones. Ann. Rep. NMR Spectrosc. 30, 231-475.

Cáceres A. (1996), Plantas de uso medicinal en Guatemala (Girón L. M. and Cáceres A. eds.). Editorial Universitaria, Guatemala, p. 89-90.

Clewell A. F. (1975), Las compuestas de Honduras. Ceiba 19, 119-244.

King R. M. and Robinson H. (1987), The genera of the Eupatorieae (Asteraceae). Monographs in Systematic Botany 22, $1-581$.

King R. M. and Robinson H. (1970), Studies in the Eupatorieae (Compositae). XIX. New combinations in Ageratina. Phytologia 19, 208-229.

Mahato S. B. and Kundu A. P. (1994), ${ }^{13}$ C NMR spectra of pentacyclic triterpenoids - a compilation and some salient features. Phytochemistry 37, 1517-1575.
Medinilla J. C. (1978), Aspectos de la medicina popular en el area rural de Guatemala. Guatemala Indigena 13, 13 (appendix).

Morton J. F. (1981), Atlas of medicinal plants of Middle America: Bahamas to Yucatan, Charles C. Thomas: Springfield, Illinois, USA, p. 933.

Nash D. L. and Williams L. O. (1976), Flora of Guatemala. Fieldiana: Botany 24, Part XII, 96.

Ober A. G., Quijano L. and Fischer N. H. (1984), Eudesmanolides, trichomatolides $\mathrm{B}-\mathrm{E}$, and a heliangolide from Calea trichomata. Phytochemistry 23, 1439-1443.

Romo J., Rios T. and Quijano L. (1968), Ligustrin, a guaianolide isolated from E. ligustrinum DC. Tetrahedron 24, 6087-6091.

Seaman F. C. (1982), Sesquiterpene lactones as taxonomic characters in the Asteraceae. Bot. Rev. 48, $121-551$.

Tamayo-Castillo G., Jakupovic J., Bohlmann F., Rojas A., Castro V. and King R. M. (1988), Germacranolides and other constituents from Ageratina species. Phytochemistry 27, 2893-2897. 